\title{
STEADY STATE SOLUTION FOR ELECTROCHEMICAL PROCESSES WITH MULTIPLE REACTING SPECIES
}

\author{
BY \\ XUN YU \\ Department of Mathematics, University of Connecticut, Storrs
}

Abstract. At steady state, the dissociation-association electrochemical reactions in a dilute solution are governed by the nondimensional equations

$$
\begin{gathered}
\frac{\partial}{\partial x}\left(d_{i} \frac{\partial u_{i}}{\partial x}+d_{i} e_{i} \frac{\partial \phi}{\partial x} u_{i}\right)+R_{i}=0, \quad i=1, \ldots, m, 0 \leq x \leq 1 \\
\sum_{i=1}^{m} e_{i} u_{i}=0
\end{gathered}
$$

where $d_{i}, e_{i}$, and $u_{i}$ are the diffusion coefficient, charge, and concentration of the $i$ th species, respectively. $\phi$ is the electric potential of the solution. $R_{i}$ is the net source of the $i$ th species in the solution which includes the external input or production due to the reactions in the solution. The extra electroneutrality condition $\sum_{i=1}^{m} e_{i} u_{i}=0$ determines the electric potential $\phi$. This system of nonlinear differential equations is subject to the nonlinear boundary conditions modeling the actual electrode kinetics. We prove the existence of the solution to this system and construct an iterative numerical algorithm to compute the solution. Numerical results are also presented in the paper.

1. Introduction. In the previous paper ([5]), the existence of a steady state solution to an electrochemical model that involves one species in a dissociation-association reaction at the boundary electrodes was proved. We shall examine a more general model in this paper where multiple species undergo the dissociation-association reactions at the boundary electrodes and prove the existence of the steady state solution for this model.

The flux of each dissolved species in a dilute solution is given by ([5])

$$
\mathbf{N}_{i}=-z_{i} \Omega_{i} F u_{i} \nabla \phi-d_{i} \nabla u_{i}+u_{i} \mathbf{v}
$$


Here $u_{i}, d_{i}$ are the concentration and the diffusion coefficient of the $i$ th species, respectively. $\phi$ is the electrostatic potential of the solution whose gradient is the negative of the electric field. $z_{i}$ is the number of proton charges carried by an ion. $\Omega_{i}$ is the mobility which denotes the average velocity of species $i$ in the solution when acted upon by a force. $F$ is Faraday's constant and $\mathbf{v}$ is the bulk velocity of the solution. The three terms $-z_{i} \Omega_{i} F u_{i} \nabla \phi,-d_{i} \nabla u_{i}$, and $u_{i} \mathbf{v}$ in the definition of $\mathbf{N}_{i}$ represent three mechanisms of mass transfer: migration of a charged species in an electric field, molecular diffusion due to a concentration gradient, and convection due to the bulk motion of the medium. In the one-dimensional case with rigid boundary, $\mathbf{v}=0$ in the above equation if we assume that the electrolyte is incompressible. Hence,

$$
\mathbf{N}_{i}=-z_{i} \Omega_{i} F u_{i} \frac{\partial \phi}{\partial x}-d_{i} \frac{\partial u_{i}}{\partial x} .
$$

The material balance for each species in the solution can be represented by

$$
\frac{\partial u_{i}}{\partial t}=-\nabla \cdot \mathbf{N}_{i}+R_{i}
$$

where $R_{i}$ is the net source of the $i$ th species, which may include external input or production due to the chemical reactions in the bulk of the solution.

The Nernst-Einstein equation $d_{i}=R T \Omega_{i}$ ([6], p. 95) provides a relationship between the diffusion coefficient and the mobility of a species. Here $R>0$ is the universal gas constant and $T$ is the absolute temperature. The pressure and thermal effects are not considered in our model.

Finally, the electrolyte must be electrically neutral everywhere, i.e.,

$$
\sum_{i} z_{i} u_{i}=0
$$

Therefore, the governing equations after non-dimensionalization become

$$
\begin{gathered}
\frac{\partial u_{i}}{\partial t}=\frac{\partial}{\partial x}\left(d_{i} \frac{\partial u_{i}}{\partial x}+d_{i} e_{i} \frac{\partial \phi}{\partial x} u_{i}\right)+R_{i}, \\
\sum_{i} e_{i} u_{i}=0
\end{gathered}
$$

where $e_{i}=z_{i} F /(R T)$ is the charge (in fact, $e_{i}$ are multiplies of $z_{i}$, we still call it the charge of species $i$ for simplicity) of the $i$ th species. Both $u_{i}$ and $\phi$ are unknowns.

In the following sections, we shall prove the existence of a steady state solution with nonlinear, nonlocal boundary conditions. Finally, a numerical algorithm is constructed to compute the numerical solution for the model and some of the numerical results are presented. 


\section{Model of multiple reacting species.}

2.1. Description of the model. Let us consider an electrochemical model in which some species with negative charge are consumed at the anode and some species with positive charge are destroyed at the cathode. We assume that once the species react at the electrodes, they never return to the solution again, for example, by becoming gases and escaping or by becoming metal and forming deposits on the surface of the electrode. The electrolyte contains many inert species which do not react at the electrodes. The reacting species are continuously replenished by an external source during the process (see Fig. 1). After an external electric power is applied to the electrodes, reacting species with positive charge in the solution move to the cathode depositing there and those reacting species with negative charge in the solution go to the anode reacting there. The system will attain steady state with the reaction rate at the electrodes being determined by the supply of the reacting species.

2.2. The governing equations. Suppose there are $m$ species in the model, the first $n$ species are reacting and the remaining $m-n$ species are inert. Without loss of generality, we assume that the first $p$ species among those $n$ reacting species have negative charge, i.e., $e_{i}<0, i=1, \ldots, p$. Then, $n-p$ reacting species have positive charge, i.e., $e_{i}>0, i=p+1, \ldots, n$. The rest of the species in the solution are inert which may be of positive or negative charge, i.e., there is no restriction on the sign of $e_{i}, i=n+1, \ldots, m$.

When the system reaches its equilibrium state, the fluxes of the reacting species with negative charge and the reacting species with positive charge satisfy the

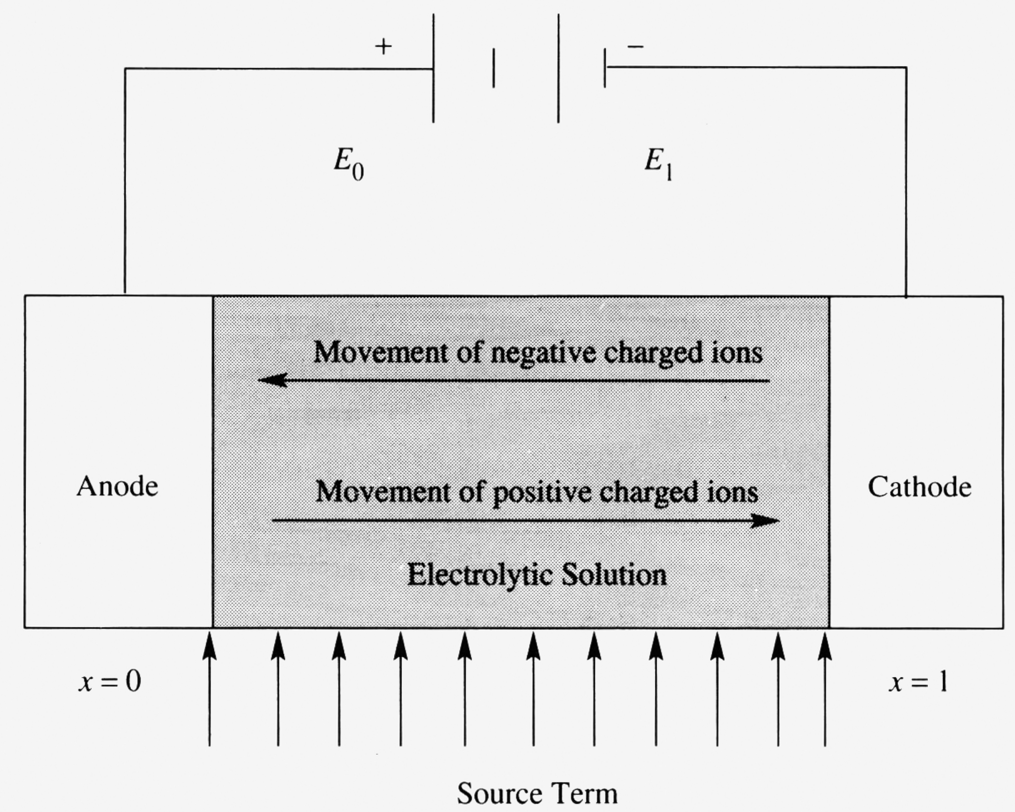

Fig. 1. Model of multiple reacting species 
equations

$$
\frac{d \mathbf{N}_{i}}{d x}=-\frac{r_{i}(x)}{e_{i}}, \quad i=1, \ldots, p
$$

and

$$
\frac{d \mathbf{N}_{i}}{d x}=+\frac{r_{i}(x)}{e_{i}}, \quad i=p+1, \ldots, n,
$$

respectively. Here $r_{i}(x) \in C[0,1], i=1, \ldots, n$, is the rate of addition of the compound composed of reacting species; so $r_{i}(x)>0, i=1, \ldots, n$. The compound has to be electrically neutral, which accounts for the form of the source term in each of the above equations. Division of $r_{i}(x)$ by $e_{i}, i=1, \ldots, n$, gives the rate of addition of the corresponding reacting species with positive and negative charge respectively. For example, for each mole of the compound $\mathrm{CaCl}_{2}$ added to the electrolyte, it gives 1 mole of $\mathrm{Ca}^{++}$ion and 2 moles of $\mathrm{Cl}^{-}$ion. Since the inert species in the solution do not participate in the electrochemical reactions at the electrodes, the fluxes of the inert species are always zero in the steady state. Moreover, the total concentration of each inert species is conserved during the steady state, and hence can be specified. Therefore, we have the following equations for the inert species:

$$
\mathbf{N}_{i}=0, \quad \int_{0}^{1} u_{i}(x) d x=c_{i}>0, \quad i=n+1, \ldots, m .
$$

The known constant $c_{i}>0, i=n+1, \ldots, m$ is the total concentration of the $i$ th inert species in the electrolyte.

$$
\mathbf{N}_{i}=-\left[d_{i} \frac{d u_{i}}{d x}+d_{i} e_{i} \frac{d \phi}{d x} u_{i}\right], \quad i=1, \ldots, m
$$

is the flux of the $i$ th species in the solution. Finally, the electroneutrality constraint

$$
\sum_{i=1}^{m} e_{i} u_{i}(x)=0
$$

has to be obeyed everywhere in the electrolyte.

REMARK. Some $r_{i}(x)$ may be equal to the sum of other $r_{i}(x)$ depending on the reacting species and the supply of the reacting species from the external source. For example, if $\mathrm{CaCl}_{2}, \mathrm{NaCl}$, and $\mathrm{ZnCl}_{2}$ are supplied through the source into the system in which $\mathrm{Ca}^{++}, \mathrm{Na}^{+}, \mathrm{Zn}^{++}$, and $\mathrm{Cl}^{-}$ions are reacting species, let $u_{1}(x)$ be the concentration of $\mathrm{Cl}^{-}$ion and $u_{2}(x), u_{3}(x)$, and $u_{4}(x)$ be the concentrations of $\mathrm{Ca}^{++}, \mathrm{Na}^{+}$, and $\mathrm{Zn}^{++}$ions, respectively. Then the governing equations are

$$
\begin{aligned}
& \frac{d N_{1}}{d x}=-\frac{r(x)}{e_{1}}, \\
& \frac{d N_{2}}{d x}=\frac{r_{2}(x)}{e_{2}}, \\
& \frac{d N_{3}}{d x}=\frac{r_{3}(x)}{e_{3}}, \\
& \frac{d N_{4}}{d x}=\frac{r_{4}(x)}{e_{4}},
\end{aligned}
$$


where $r(x)=r_{2}(x)+r_{3}(x)+r_{4}(x)$ and $r_{2}(x), r_{3}(x)$, and $r_{4}(x)$ are the sources of material $\mathrm{CaCl}_{2}, \mathrm{NaCl}$, and $\mathrm{ZnCl}$, respectively.

2.3. The boundary conditions. We have to supplement the governing system of algebraic and differential equations (1)-(4) with boundary conditions. Intuitively, at steady state, the fluxes of positively-charged species in the solution are zero at the anode since they do not react there. The fluxes at the cathode are determined by the reaction rates which depend on the concentrations of the reacting species and the surface overpotential at the cathode ([5]). Similar statements can be made about the fluxes of negatively-charged species at the two electrodes. Motivated by typical electrode kinetics expressions in electrochemical literature ([6], p. 111), and after taking into account the sign convention of positive flux direction, the boundary conditions for the negatively-charged reacting species can be represented by

$$
\left.\mathbf{N}_{i}\right|_{x=1}=0,\left.\quad \mathbf{N}_{i}\right|_{x=0}=-f_{i}\left(u_{i}(0)\right) g_{i}(\phi(0)), \quad i=1, \ldots, p .
$$

Similarly, the fluxes of the positively-charged reacting species must satisfy

$$
\left.\mathbf{N}_{i}\right|_{x=0}=0,\left.\quad \mathbf{N}_{i}\right|_{x=1}=+f_{i}\left(u_{i}(1)\right) g_{i}(\phi(1)), \quad i=p+1, \ldots, n
$$

where $f_{i}(u)$ and $g_{i}(v), i=1,2, \ldots, n$, are known $C^{1}$ functions that possess the following properties:

$$
\begin{gathered}
f_{i}^{\prime}(u)>0 \text { if } u>0, f_{i}(0)=0 \quad \text { and } f_{i}(u) \rightarrow \infty \text { as } u \rightarrow \infty, i=1,2, \ldots, n, \\
g_{i}^{\prime}(v)<0, g_{i}\left(E_{0}\right)=0, \quad \text { and } g_{i}(v) \rightarrow \infty \text { as } v \rightarrow-\infty, i=1, \ldots, p, \\
g_{i}^{\prime}(v)>0, g_{i}\left(E_{1}\right)=0, \quad \text { and } g_{i}(v) \rightarrow \infty \text { as } v \rightarrow \infty, i=p+1, \ldots, n,
\end{gathered}
$$

where $E_{1}<E_{0}$ are the external electric potentials applied to the electrodes. Condition (7) indicates that the higher the reactant concentration is at the electrode, the faster is the reaction, and when the reactant concentration is zero at the electrode, there cannot be any reactions. Condition (8) states that the larger the solution potential $\phi(x)$ is at the anode, the smaller is the surface overpotential $\left(E_{0}-\phi(0)\right.$ at the anode and $\phi(1)-E_{1}$ at the cathode, cf. [5]), leading to slower reaction. If there is no surface overpotential at the anode (i.e., $\phi(0)=E_{0}$ ), there will be no electrochemical reactions. Condition (9) has an analogous interpretation. Finally, it is noted that $g_{i}<0$ if $v>E_{0}$ for $i=1, \ldots, p$ and $g_{i}<0$ if $v<E_{1}$ for $i=p+1, \ldots, n$.

2.4. Existence of the steady state solution. In this section, we shall prove the following theorem.

TheOREM 2.1. There exists a solution $\phi(x)$ and $u_{i}(x), i=1, \ldots, m$, to the equations (1), (2), (3), and (4) subject to the boundary conditions (5) and (6) where $f$ and $g$ satisfy conditions (7), (8), and (9). Moreover, the solution satisfies $E_{1}<\phi(x)<E_{0}$ and $u_{i}(x)>0, i=1, \ldots, m$, for all $x \in[0,1]$.

First, we will simplify equations (1), (2), (3), (4) and boundary conditions (5), (6). Integrating (1) from $x$ to 1 using (5) and integrating (2) from 0 to $x$ applying (6) 
respectively, we have

$$
\begin{gathered}
-\mathbf{N}_{i}=d_{i} \frac{d u_{i}(x)}{d x}+d_{i} e_{i} \frac{d \phi(x)}{d x} u_{i}(x)=-\frac{p_{i}(x)}{e_{i}}, \quad i=1, \ldots, p, \\
-\mathbf{N}_{i}=d_{i} \frac{d u_{i}(x)}{d x}+d_{i} e_{i} \frac{d \phi(x)}{d x} u_{i}(x)=-\frac{p_{i}(x)}{e_{i}}, \quad i=p+1, \ldots, n
\end{gathered}
$$

where

$$
p_{i}(x)= \begin{cases}\int_{x}^{1} r_{i}(t) d t>0, & \text { if } i=1, \ldots, p, \\ \int_{0}^{x} r_{i}(t) d t>0, & \text { if } i=p+1, \ldots, n\end{cases}
$$

for all $x \in[0,1]$. Therefore, (5) and (6) become $f_{i}\left(u_{i}(0)\right) g_{i}(\phi(0))=a_{i}>0$ for $i=1, \ldots, p$ and $f_{i}\left(u_{i}(1)\right) g_{i}(\phi(1))=a_{i}>0$ for $i=p+1, \ldots, n$ respectively, where

$$
a_{i}= \begin{cases}-\frac{1}{e_{i}} \int_{0}^{1} r_{i}(x) d x>0, & \text { if } i=1, \ldots, p, \\ \frac{1}{e_{i}} \int_{0}^{1} r_{i}(x) d x>0, & \text { if } i=p+1, \ldots, n .\end{cases}
$$

We can then consider the equivalent system

$$
\begin{gathered}
d_{i} \frac{d u_{i}(x)}{d x}+d_{i} e_{i} \frac{d \phi(x)}{d x} u_{i}(x)=-\frac{1}{e_{i}} p_{i}(x), \quad i=1,2, \ldots, n, \\
\mathbf{N}_{i}=0, \quad \int_{0}^{1} u_{i}(x) d x=c_{i}>0, \quad i=n+1, \ldots, m, \\
\sum_{i=1}^{m} e_{i} u_{i}(x)=0, \\
f_{i}\left(u_{i}(0)\right) g_{i}(\phi(0))=a_{i}>0, \quad i=1, \ldots, p, \\
f_{i}\left(u_{i}(1)\right) g_{i}(\phi(1))=a_{i}>0, \quad i=p+1, \ldots, n,
\end{gathered}
$$

where $e_{i}<0, i=1, \ldots, p$, and $e_{i}>0, i=p+1, \ldots, n$. To prove Theorem 2.1, it suffices to show that system (10)-(14) has a solution such that $E_{1}<\phi(x)<E_{0}$ and $u_{i}(x)>0, i=1, \ldots, m$, for all $x \in[0,1]$.

Now multiplying each equation in (10) and (11) by $e_{i} / d_{i}, i=1, \ldots, m$, respectively, summing over $i, i=1, \ldots, m$, and using (12), we get

$$
\frac{d \phi(x)}{d x} \sum_{i=1}^{m} e_{i}^{2} u_{i}(x)=-P(x)
$$

where $P(x)=\sum_{i=1}^{n} \frac{1}{d_{i}} p_{i}(x)>0$.

Let

$$
S=\left\{\phi(x) \in C^{1}[0,1] \mid-M \leq \frac{d \phi(x)}{d x} \leq 0, E_{1} \leq \phi(x) \leq E_{0}\right\}
$$

where $M$ is a positive constant to be determined later. Then given any $\phi \in S$, all $u_{i}$ can be solved in terms of $\phi(x)$ from (10) and (11), that is,

$$
u_{i}(x)=e^{-e_{i}(\phi(x)-\phi(0))}\left[-\int_{0}^{x} e^{e_{i}(\phi(s)-\phi(0))} \frac{p_{i}(s)}{d_{i} e_{i}} d s+k_{i}\right], \quad i=1, \ldots, n,
$$


and

$$
u_{i}(x)=\frac{c_{i} e^{-e_{i} \phi(x)}}{\int_{0}^{1} e^{-e_{i} \phi(x)} d x}, \quad i=n+1, \ldots, m,
$$

where $k_{i}=u_{i}(0)$ is an unknown constant, $i=1,2, \ldots, n$.

Note that we can eliminate $\phi(0)$ in (13) and $\phi(1)$ in (14) to get

$$
g_{1}^{-1}\left(\frac{a_{1}}{f_{1}\left(u_{1}(0)\right)}\right)=g_{i}^{-1}\left(\frac{a_{i}}{f_{i}\left(u_{i}(0)\right)}\right), \quad i=1, \ldots, p
$$

and

$$
g_{p+1}^{-1}\left(\frac{a_{p+1}}{f_{p+1}\left(u_{p+1}(1)\right)}\right)=g_{i}^{-1}\left(\frac{a_{i}}{f_{i}\left(u_{i}(1)\right)}\right), i=p+1, \ldots, n,
$$

using the monotonicity of functions $f_{i}$ and $g_{i}, i=1, \ldots, n$. It can be seen from (18) that if $u_{1}(0)=k_{1}>0$ is given, then $u_{i}(0)=k_{i}>0, i=2, \ldots, p$, can be solved in terms of $u_{1}(0)=k_{1}$. Hence, each $u_{i}(x)>0, i=2, \ldots, p$, can be calculated from (16). Similarly, if

$$
k_{p+1}=u_{p+1}(0)>\int_{0}^{1} e^{e_{p+1}(\phi(s)-\phi(0))} \frac{p_{p+1}(s)}{d_{p+1} e_{p+1}} d s>0
$$

is given, $u_{i}(1)>0, i=p+2, \ldots, n$, can be found from (19). Moreover, each $u_{i}(x)>0, i=p+2, \ldots, n$, for all $x \in[0,1]$ because of the fact that

$$
u_{i}(1)=e^{-e_{i}(\phi(1)-\phi(0))}\left[-\int_{0}^{1} e^{e_{i}(\phi(s)-\phi(0))} \frac{p_{i}(s)}{d_{i} e_{i}} d s+k_{i}\right]>0
$$

implies that

$$
k_{i}>\int_{0}^{1} e^{e_{i}(\phi(s)-\phi(0))} \frac{p_{i}(s)}{d_{i} e_{i}} d s>\int_{0}^{x} e^{e_{i}(\phi(s)-\phi(0))} \frac{p_{i}(s)}{d_{i} e_{i}} d s>0, \quad i=p+2, \ldots, n,
$$

for all $x \in[0,1]$. Therefore, the constants $k_{i}, i=1, \ldots, p, p+1, \ldots, n$, are dependent on two constants only, $k_{1}$ and $k_{p+1}$.

We will construct a map $T: S \rightarrow C^{1}$ in the following way. Let $\phi \in S$ and define $u_{i}, i=1, \ldots, m$, by (16) and (17) up to the constants $k_{1}=u_{1}(0)$ and $k_{p+1}=u_{p+1}(0)$. Then $T \phi$ is defined to be the solution to (15) such that (13) is satisfied for $i=1,(14)$ is satisfied for $i=p+1$, and (12) is satisfied at $x=0$. Lemmas 2.1 and 2.2 are used to justify the validity of the definition of $T$ and Lemma 2.3 shows that $T$ is compact and maps $S \rightarrow S$. Hence Schauder's fixed point theorem applies.

Let

$$
m_{0}=\max _{\substack{1 \leq i \leq n \\ 0 \leq x \leq 1}} r_{i}(x)
$$

Note that $e_{i}<0$ for $i=1, \ldots, p, e_{i}>0$ for $i=p+1, \ldots, n, \phi(x)$ is nonincreasing and $E_{1} \leq \phi \leq E_{0}$. It can be checked from (16) that if $k_{i}>0, i=1, \ldots, p$,

$$
e^{\left|e_{i}\right|\left(E_{1}-E_{0}\right)} k_{i} \leq u_{i}(x) \leq e^{\left|e_{i}\right|\left(E_{0}-E_{1}\right)} \frac{m_{0}}{d_{i}\left|e_{i}\right|}+k_{i}=m_{i}+k_{i}, \quad i=1, \ldots, p
$$


where $m_{i}=e^{\left|e_{i}\right|\left(E_{0}-E_{1}\right)} m_{0} /\left(d_{i}\left|e_{i}\right|\right)>0$ for $i=1, \ldots, p$. Since

$$
0<\int_{0}^{x} e^{e_{i}(\phi(s)-\phi(0))} \frac{p_{i}(s)}{d_{i} e_{i}} d s<\int_{0}^{1} e^{e_{i}(\phi(s)-\phi(0))} \frac{p_{i}(s)}{d_{i} e_{i}} d s, \quad i=p+1, \ldots, n
$$

for all $x \in[0,1]$, if

$$
k_{i}>\int_{0}^{1} e^{e_{i}(\phi(s)-\phi(0))} \frac{p_{i}(s)}{d_{i} e_{i}} d s>0, \quad i=p+1, \ldots, n,
$$

then

$$
0<u_{i}(x) \leq e^{\left|e_{i}\right|\left(E_{0}-E_{1}\right)} k_{i}=m_{i} k_{i}, \quad i=p+1, \ldots, n
$$

where $m_{i}=e^{\left|e_{i}\right|\left(E_{0}-E_{1}\right)}>0$ for $i=p+1, \ldots, n$. Finally,

$$
0<c_{i} e^{\left|e_{i}\right|\left(E_{1}-E_{0}\right)} \leq u_{i}(x) \leq c_{i} e^{\left|e_{i}\right|\left(E_{0}-E_{1}\right)}, \quad i=n+1, \ldots, m .
$$

Inequalities (20), (21), and (22) will be used to show that $T$ is compact in Lemma 2.3.

Now, differentiating both sides of (18) with respect to $k_{1}$, we have

$$
\left(g_{1}^{-1}\right)^{\prime} \frac{a_{1}}{f_{1}^{2}} f_{1}^{\prime}=\left(g_{i}^{-1}\right)^{\prime} \frac{a_{i}}{f_{i}^{2}} f_{i}^{\prime} \frac{d k_{i}}{d k_{1}}, \quad i=1, \ldots, p
$$

which implies, using (7) and (8), that

$$
\frac{d k_{i}}{d k_{1}}=\frac{a_{1}\left(g_{1}^{-1}\right)^{\prime} f_{i}^{2} f_{1}^{\prime}}{a_{i}\left(g_{i}^{-1}\right)^{\prime} f_{1}^{2} f_{i}^{\prime}}>0, \quad i=1, \ldots, p .
$$

Similarly, differentiating both sides of (19) with respect to $k_{p+1}$, we have

$$
\left(g_{p+1}^{-1}\right)^{\prime} \frac{a_{p+1}}{f_{p+1}^{2}} f_{p+1}^{\prime} e^{-e_{p+1}(\phi(1)-\phi(0))}=\left(g_{i}^{-1}\right)^{\prime} \frac{a_{i}}{f_{i}^{2}} f_{i}^{\prime} e^{-e_{i}(\phi(1)-\phi(0))} \frac{d k_{i}}{d k_{p+1}},
$$

$i=p+2, \ldots, n$. Hence,

$$
\frac{d k_{i}}{d k_{p+1}}=e^{\left(e_{i}-e_{p+1}\right)(\phi(1)-\phi(0))} \frac{a_{p+1} f_{p+1}^{\prime}\left(g_{p+1}^{-1}\right)^{\prime} f_{i}^{2}}{a_{i} f_{i}^{\prime}\left(g_{i}^{-1}\right)^{\prime} f_{p+1}^{2}}>0, \quad i=p+1, \ldots, n,
$$

considering (7) and (9). (23) and (24) are used to prove the following lemma.

LemMA 2.1. Given $a_{1}>0, a_{p+1}>0$, and $\phi \in S$, we construct $u_{i}, i=1, \ldots, m$, by (16), (17), (18), and (19). Then there exists a unique solution $k_{1}>0, k_{p+1}>M_{0}$, and $E_{1}<\gamma<E_{0}$ such that the following equations are satisfied:

$$
\begin{gathered}
f_{1}\left(k_{1}\right) g_{1}(\gamma)=a_{1}>0, \\
f_{p+1}\left(u_{p+1}(1)\right) g_{p+1}\left(\gamma-\int_{0}^{1} \frac{P(x)}{h\left(x, k_{1}, k_{p+1}\right)} d x\right)=a_{p+1}>0, \\
\sum_{i=1}^{m} e_{i} u_{i}(0)=\sum_{i=1}^{n} e_{i} k_{i}+\sum_{i=n+1}^{m} e_{i} c_{i} \frac{e^{-e_{i} \phi(0)}}{\int_{0}^{1} e^{-e_{i} \phi(x)} d x}=0 .
\end{gathered}
$$


Here $u_{p+1}(1)$ is determined by (16). $M_{0}$ is a positive constant that depends on $\phi, r_{i}, f_{i}, g_{i}, c_{i}, e_{i}, d_{i}$, and

$$
h\left(x, k_{1}, k_{p+1}\right)=\sum_{i=1}^{n} e_{i}^{2} u_{i}^{+}(x)+\sum_{i=n+1}^{m} e_{i}^{2} u_{i}(x)
$$

where

$$
u_{i}^{+}(x)=\left\{\begin{array}{ll}
u_{i}(x) & \text { if } u_{i}(x)>0 \\
0 & \text { if } u_{i}(x) \leq 0
\end{array} \quad i=1, \ldots, n .\right.
$$

Proof. Let $F_{1}\left(k_{1}\right)=\sum_{i=1}^{p} e_{i} k_{i}$ and $F_{2}\left(k_{p+1}\right)=\sum_{i=p+1}^{n} e_{i} k_{i}$. Then

$$
\frac{d F_{1}}{d k_{1}}=\sum_{i=1}^{p} e_{i} \frac{d k_{i}}{d k_{1}}<0
$$

because $e_{i}<0$ for $i=1, \ldots, p$ and (23). Similarly,

$$
\frac{d F_{2}}{d k_{p+1}}=\sum_{i=p+1}^{n} e_{i} \frac{d k_{i}}{d k_{p+1}}>0
$$

since each $\frac{d k_{i}}{d k_{p+1}}>0$ and $e_{i}>0$ for $i=p+1, \ldots, n$. Now (27) can be written as

$$
F_{1}\left(k_{1}\right)+F_{2}\left(k_{p+1}\right)+\sum_{i=n+1}^{m} e_{i} c_{i} \frac{e^{-e_{i} \phi(0)}}{\int_{0}^{1} e^{-e_{i} \phi(x)} d x}=0 .
$$

We will derive the relationship between variables $\gamma$ and $k_{p+1}$ and plot $\gamma$ as a function of $k_{p+1}$ for (25) and (26) to obtain the solution.

Differentiating both sides of $(30)$ with respect to $k_{p+1}$, we have

$$
\frac{d F_{1}}{d k_{1}} \frac{d k_{1}}{d k_{p+1}}+\frac{d F_{2}}{d k_{p+1}}=0
$$

which implies, using (28) and (29), that

$$
\frac{d k_{1}}{d k_{p+1}}=-\frac{\frac{d F_{2}}{d k_{p+1}}}{\frac{d F_{1}}{d k_{1}}}>0
$$

Next, differentiating both sides of (25) with respect to $k_{p+1}$, we get

$$
f_{1}^{\prime}\left(k_{1}\right) \frac{d k_{1}}{d k_{p+1}} g_{1}(\gamma)+f_{1}\left(k_{1}\right) g_{1}^{\prime}(\gamma) \frac{d \gamma}{d k_{p+1}}=0
$$

which implies that $\frac{d \gamma}{d k_{p+1}}>0$ using (7), (8), and (31) if $\gamma<E_{0}$ and $k_{1}>0$. Note that there exists a number $M_{1}$ (may be positive or negative) such that

$$
F_{2}\left(M_{1}\right)+\sum_{i=n+1}^{m} e_{i} c_{i} \frac{e^{-e_{i} \phi(0)}}{\int_{0}^{1} e^{-e_{i} \phi(x)} d x}=0 .
$$




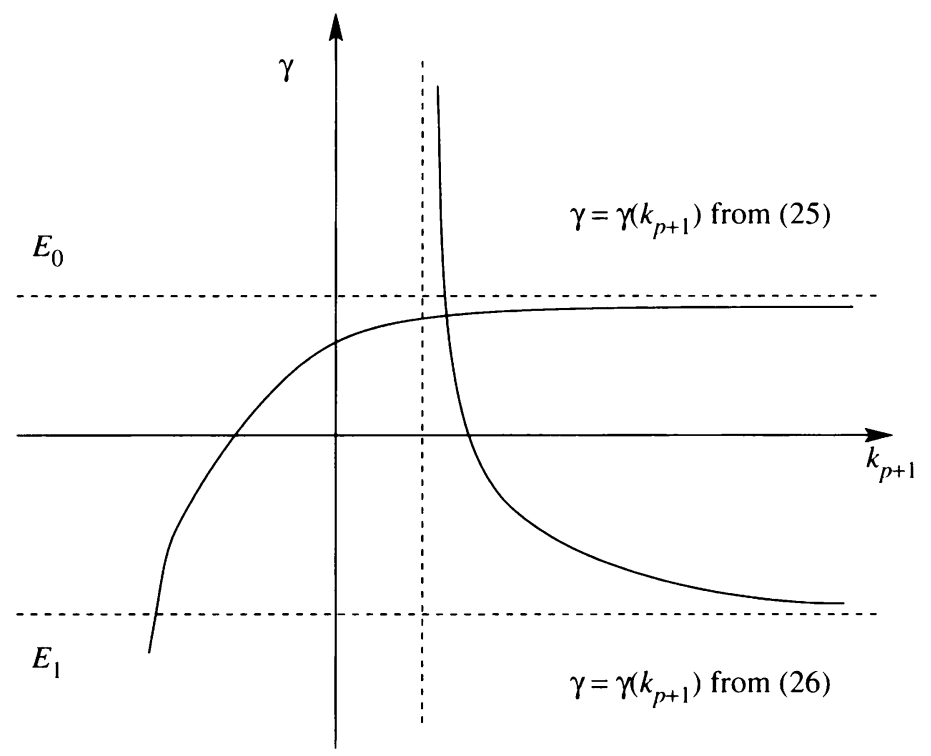

FIG. 2. Graphs of (25) and (26)

So as $k_{p+1} \searrow M_{1}, F_{1}\left(k_{1}\right) \rightarrow 0$, which implies that $k_{i} \searrow 0, i=1, \ldots, p$ and hence $k_{1} \searrow 0$. (25) then implies that $\gamma \searrow-\infty$. Similarly $k_{p+1} \rightarrow+\infty$ implies that $k_{1} \rightarrow+\infty$ by (30) and (31), and so $\gamma / E_{0}$ from below by (25).

It is noted that if

$$
k_{p+1}>\int_{0}^{1} e^{e_{p+1}(\phi(s)-\phi(0))} \frac{p_{p+1}(s)}{d_{p+1} e_{p+1}} d s>0,
$$

then $f_{p+1}\left(u_{p+1}(1)\right)>0$ and $h\left(x, k_{1}, k_{p+1}\right)>0$. We can solve $\gamma$ from (26) such that

$$
\gamma>E_{1}+\int_{0}^{1} \frac{P(x)}{h\left(x, k_{1}, k_{p+1}\right)} d x>E_{1} .
$$

Now, differentiating both sides of $(26)$ with respect to $k_{p+1}$, we end up with $e^{-e_{p+1}(\phi(1)-\phi(0))} f_{p+1}^{\prime} g_{p+1}+f_{p+1} g_{p+1}^{\prime} \cdot\left(\frac{d \gamma}{d k_{p+1}}-\frac{d}{d k_{p+1}}\left(\int_{0}^{1} \frac{P(x)}{h\left(x, k_{1}, k_{p+1}\right)} d x\right)\right)=0$.

It can be checked that

$$
\frac{d}{d k_{p+1}}\left(\int_{0}^{1} \frac{P(x)}{h\left(x, k_{1}, k_{p+1}\right)} d x\right)<0 .
$$

Applying (7) and (9), we have $d \gamma / d k_{p+1}<0$ if

$$
k_{p+1}>\int_{0}^{1} e^{e_{p+1}(\phi(s)-\phi(0))} \frac{p_{p+1}(s)}{d_{p+1} e_{p+1}} d s
$$

and $\gamma>E_{1}$. It can be seen that as

$$
k_{p+1} \searrow \int_{0}^{1} e^{e_{p+1}(\phi(s)-\phi(0))} \frac{p_{p+1}(s)}{d_{p+1} e_{p+1}} d s>0,
$$


$\gamma \nearrow+\infty$ by (26), and similarly, as $k_{p+1} \nearrow+\infty, \gamma \searrow E_{1}$ from above. Therefore, the graph of (25) intersects that of (26) at exactly one point in the region

$$
k_{p+1}>M_{0}=\max \left(\int_{0}^{1} e^{e_{p+1}(\phi(s)-\phi(0))} \frac{p_{p+1}(s)}{d_{p+1} e_{p+1}} d s, M_{1}\right)>0
$$

and $E_{1}<\gamma<E_{0}$ (see Fig. 2). Note that if $k_{1}>0$ and $k_{p+1}>M_{0}$, we have $u_{i}^{+}(x)=u_{i}(x), i=1, \ldots, n$. Hence system (25)-(27) has a unique solution $k_{1}>$ $0, k_{p+1}>M_{0}>0$, and $E_{1}<\gamma<E_{0}$. Moreover, $h\left(x, k_{1}, k_{p+1}\right)$ can be replaced with $\sum_{i=1}^{m} e_{i}^{2} u_{i}(x)$ in (26).

LemMA 2.2. Given $\phi \in S, a_{1}>0$, and $a_{p+1}>0$, the following system

$$
\begin{gathered}
\frac{d y(x)}{d x}=-\frac{P(x)}{H(x)}, \\
f_{1}\left(k_{1}\right) g_{1}(y(0))=a_{1}>0, \\
f_{p+1}\left(u_{p+1}(1)\right) g_{p+1}(y(1))=a_{p+1}>0, \\
\sum_{i=1}^{m} e_{i} u_{i}(0)=\sum_{i=1}^{n} e_{i} k_{i}+\sum_{i=n+1}^{m} e_{i} c_{i} \frac{e^{-e_{i} \phi(0)}}{\int_{0}^{1} e^{-e_{i} \phi(x)} d x}=0,
\end{gathered}
$$

where $H(x)=\sum_{i=1}^{m} e_{i}^{2} u_{i}(x)$, has a unique solution $y(x) \in\left[E_{1}, E_{0}\right], k_{1}>0$ and $k_{p+1}>M_{0}>0$.

Proof. Integrating (32) from 0 to $x$, we have

$$
y(x)=y(0)-\int_{0}^{x} \frac{P(s)}{H(s)} d s .
$$

By Lemma 2.1, system (33)-(35) with $y(1)$ replaced by (36) has a unique solution $k_{1}>0, k_{p+1}>M_{0}>0$ and $E_{1}<y(0)<E_{0}$. Furthermore, $y(1)>E_{1}$, for if $y(1) \leq E_{1}$, then $g_{p+1}(y(1)) \leq 0$ implies that $f_{p+1} g_{p+1} \leq 0$ which contradicts (34). Hence, the system (32)-(35) has a unique solution such that $k_{1}>0, k_{p+1}>M_{0}>0$, and $y(x) \in\left[E_{1}, E_{0}\right]$.

We now define a map $y(x)=T \phi(x)$ to be the solution to the boundary value problem (32)-(34) satisfying condition (35).

Lemma 2.3. $T: S \rightarrow S$ is continuous and $T$ is compact when the constant $M$ in the definition of $S$ is sufficiently large.

Proof. The continuity of $T$ is straightforward ([5]). Recall the set $S$ defined earlier in this section. We can take

$$
\begin{aligned}
M & =\frac{m_{0}\left(\sum_{i=1}^{n} \frac{1}{d_{i}}\right)}{e_{n+1}^{2} c_{n+1} e^{\left|e_{n+1}\right|\left(E_{1}-E_{0}\right)}} \geq \frac{m_{0}\left(\sum_{i=1}^{n} \frac{1}{d_{i}}\right)}{e_{n+1}^{2} u_{n+1}(x)} \\
& \geq\left|-\frac{P(x)}{H(x)}\right|=\left|\frac{d T \phi(x)}{d x}\right|
\end{aligned}
$$


since $|P(x)| \leq m_{0}\left(\sum_{i=1}^{n} \frac{1}{d_{i}}\right)$ and $e_{n+1}^{2} c_{n+1} e^{\left|e_{n+1}\right|\left(E_{1}-E_{0}\right)} \leq e_{n+1}^{2} u_{n+1}(x)<H(x)$ from (22). Since $d T \phi(x) / d x \leq 0, T$ maps $S \rightarrow S$.

We next show that the map $T$ is compact. It can be checked from Eqs. (10) and (11), using (20), (21), and (22), that

$$
\begin{aligned}
& \left|u_{i}^{\prime}(x)\right| \leq \frac{m_{0}}{\left|e_{i}\right| d_{i}}+\left|e_{i}\right| M\left(m_{i}+k_{i}\right), \quad i=1, \ldots, p, \\
& \left|u_{i}^{\prime}(x)\right| \leq \frac{m_{0}}{\left|e_{i}\right| d_{i}}+\left|e_{i}\right| M m_{i} k_{i}, \quad i=p+1, \ldots, n, \\
& \left|u_{i}^{\prime}(x)\right| \leq\left|e_{i}\right| M c_{i} e^{\left|e_{i}\right|\left(E_{0}-E_{1}\right)}, \quad i=n+1, \ldots, m,
\end{aligned}
$$

and

$$
\left|P^{\prime}(x)\right| \leq m_{0}\left(\sum_{i=1}^{n} \frac{1}{d_{i}}\right)
$$

From (20), (21), and (22),

$$
\begin{aligned}
|H(x)| & =\left|\sum_{i=1}^{m} e_{i}^{2} u_{i}(x)\right| \\
& \leq \sum_{i=1}^{p} e_{i}^{2}\left(m_{i}+k_{i}\right)+\sum_{i=p+1}^{n} e_{i}^{2} m_{i} k_{i}+\sum_{i=n+1}^{m} e_{i}^{2} c_{i} e^{\left|e_{i}\right|\left(E_{0}-E_{1}\right)} \\
& =L+\sum_{i=1}^{p} e_{i}^{2} k_{i}+\sum_{i=p+1}^{n} e_{i}^{2} m_{i} k_{i}
\end{aligned}
$$

where

$$
L=\sum_{i=1}^{p} e_{i}^{2} m_{i}+\sum_{i=n+1}^{m} e_{i}^{2} c_{i} e^{\left|e_{i}\right|\left(E_{0}-E_{1}\right)}
$$

and

$$
\begin{aligned}
\left|\frac{d}{d x} H(x)\right| \leq & \sum_{i=1}^{p} e_{i}^{2}\left(\frac{m_{0}}{\left|e_{i}\right| d_{i}}+\left|e_{i}\right| M\left[m_{i}+k_{i}\right]\right) \\
& +\sum_{i=p+1}^{n} e_{i}^{2}\left(\frac{m_{0}}{\left|e_{i}\right| d_{i}}+\left|e_{i}\right| M m_{i} k_{i}\right)+\sum_{i=n+1}^{m}\left|e_{i}\right|^{3} M c_{i} e^{\left|e_{i}\right|\left(E_{0}-E_{1}\right)} \\
= & Q+\sum_{i=1}^{p}\left|e_{i}\right|^{3} M k_{i}+\sum_{i=p+1}^{n}\left|e_{i}\right|^{3} M m_{i} k_{i}
\end{aligned}
$$

where

$$
Q=\sum_{i=1}^{n} \frac{\left|e_{i}\right| m_{0}}{d_{i}}+\sum_{i=1}^{p}\left|e_{i}\right|^{3} M m_{i}+\sum_{i=n+1}^{m}\left|e_{i}\right|^{3} M c_{i} e^{\left|e_{i}\right|\left(E_{0}-E_{1}\right)}
$$


Thus,

$$
\begin{aligned}
\left|\frac{d^{2} T \phi(x)}{d x^{2}}\right|= & \frac{P^{\prime}(x) H(x)-P(x) H^{\prime}(x)}{[H(x)]^{2}} \\
\leq & \frac{\left(\sum_{i=1}^{n} \frac{m_{0}}{d_{i}}\right)\left(L+Q+\sum_{i=1}^{p} e_{i}^{2}\left(\left|e_{i}\right| M+1\right) k_{i}\right)}{[H(x)]^{2}} \\
& +\frac{\left(\sum_{i=1}^{n} \frac{m_{0}}{d_{i}}\right)\left(\sum_{i=p+1}^{n} e_{i}^{2} m_{i}\left(1+\left|e_{i}\right| M\right) k_{i}\right)}{[H(x)]^{2}} \\
\leq & \frac{\left(\sum_{i=1}^{n} \frac{m_{0}}{d_{i}}\right)\left(R+M_{2} \sum_{i=1}^{n} e_{i}^{2}\left(1+\left|e_{i}\right| M\right) k_{i}\right)}{[H(x)]^{2}}
\end{aligned}
$$

where $M_{2}=\max _{p+1 \leq i \leq n}\left(m_{i}, 1\right)>0$ and $R=L+Q>0$. It is bounded for all $k_{1}>0, k_{p+1}>M_{0}$. This, together with the fact that $T \phi(x), d T \phi(x) / d x$ are bounded, implies that $T$ is compact.

Proof of the theorem. By Schauder's fixed point theorem, there exists $\phi \in S$ such that $T \phi(x)=\phi(x)$ because of Lemma 2.3 and the fact that $S$ is closed and convex. Finally, Eqs. (10), (11), and (32) imply that $\sum_{i=1}^{m} e_{i} d u_{i}(x) / d x=0$. After integrating from 0 to $x$ using (35), we have that $\sum_{i=1}^{m} e_{i} u_{i}(x)=\sum_{i=1}^{m} e_{i} u_{i}(0)=0$. Hence, system (10)-(14) has a solution $u_{i}(x)>0, i=1, \ldots, m$, and $E_{1}<\phi(x)<E_{0}$ for all $x \in[0,1]$. This completes the proof of the theorem.

\section{Numerical algorithm for the steady state solution.}

3.1. Description of algorithm. This algorithm is derived based on the existence proof in the foregoing section. First, the interval $[0,1]$ in the $x$-direction is discretized uniformly. Since the solution $\phi(x)$ is monotone decreasing and lies in the interval $\left[E_{1}, E_{0}\right]$, we can give a good guess of $\phi(x)$ to start with, for example, a straight line between values $E_{1}$ and $E_{0}$. The values of the concentration of the inert species $u_{i}(x), i=n+1, \ldots, m$, can be computed by (17). If the initial values $k_{1}>0$ and $k_{p+1}>M_{0}$ are chosen, the values of all $k_{i}, i=2, \ldots, p, p+2, \ldots, n$ can be computed via (18) and (19), i.e.,

$$
\begin{aligned}
k_{i}= & f_{i}^{-1}\left(\frac{a_{i}}{g_{i}\left(g_{1}^{-1}\left(\frac{a_{1}}{f_{1}\left(k_{1}\right)}\right)\right)}\right) \text { for } i=2, \ldots, p, \\
k_{i}= & f_{i}^{-1}\left(\frac{a_{i}}{g_{i}\left(g_{p+1}^{-1}\left(\frac{a_{p+1}}{f_{p+1}\left(u_{p+1}(1)\right)}\right)\right)}\right) e^{e_{i}(\phi(1)-\phi(0))} \\
& +\int_{0}^{1} e^{e_{i}(\phi(s)-\phi(0))} \frac{1}{d_{i} e_{i}} p_{i}(s) d s \quad \text { for } i=p+2, \ldots, n
\end{aligned}
$$


where the functions $f_{i}, g_{i}$ are known and $a_{i}, i=1, \ldots, n$, can be calculated. Thus, the values of the concentration of the reacting species $u_{i}(x), i=1, \ldots, n$, can be calculated from (16). Lemma 2.1 guarantees that the system (25)-(27) has a solution in the desired region. We can now apply Newton's method to solve this nonlinear system as long as we have good initial values for $k_{1}, k_{p+1}$, and $\gamma$. Once the system (25)-(27) has been solved, the new values of $k_{1}$ and $k_{p+1}$ can be used to compute $k_{i}, i=2, \ldots, p, p+2, \ldots, n$, via (18) and (19). Then $u_{i}, i=1, \ldots, n$, is computed using (16). Finally, $\phi(x)$ is updated by

$$
\phi(x)=\gamma-\int_{0}^{x} \frac{P(s)}{\sum_{i=1}^{m} e_{i}^{2} u_{i}(s)} d s .
$$

The new value $\phi(x)$ is then used as a new initial value for $\phi(x)$, and the previous steps are repeated again until the differences between the values of $\phi(x)$ and $u_{i}, i=$ $1, \ldots, m$, at every mesh point in the successive iterations are less than a certain tolerance, say $10^{-6}$.

It is noted that the key step of the algorithm is to solve the nonlinear system (25)(27) using Newton's method in which a $3 \times 3$ Jacobian matrix $\mathbf{J}$ has to be computed. Let

$$
\begin{aligned}
F_{1} & =f_{1}\left(k_{1}\right) g_{1}(\gamma)-a_{1}=0 \\
F_{2} & =f_{p+1}\left(u_{p+1}(1)\right) g_{p+1}\left(\gamma-\int_{0}^{1} \frac{P(x)}{\sum_{i=1}^{m} e_{i}^{2} u_{i}} d x\right)-a_{p+1}=0, \\
F_{3} & =\sum_{i=1}^{m} e_{i} u_{i}(0) \\
& =\sum_{i=1}^{n} e_{i} k_{i}+\sum_{i=n+1}^{m} e_{i} c_{i} \frac{e^{-e_{i} \phi(0)}}{\int_{0}^{1} e^{-e_{i} \phi(x)} d x}=0 .
\end{aligned}
$$

The Jacobian matrix of this system is

$$
\mathbf{J}_{3 \times 3}\left(k_{1}, k_{p+1}, \gamma\right)=\left[\begin{array}{ccc}
\frac{\partial F_{1}}{\partial k_{1}} & \frac{\partial F_{1}}{\partial k_{p+1}} & \frac{\partial F_{1}}{\partial \gamma} \\
\frac{\partial F_{2}}{\partial k_{1}} & \frac{\partial F_{2}}{\partial k_{p+1}} & \frac{\partial F_{2}}{\partial \gamma} \\
\frac{\partial F_{3}}{\partial k_{1}} & \frac{\partial F_{3}}{\partial k_{p+1}} & \frac{\partial F_{3}}{\partial \gamma}
\end{array}\right] .
$$

Clearly, $\partial F_{1} / \partial k_{p+1}=0, \partial F_{3} / \partial \gamma=0$, and other elements in the Jacobian matrix 
are given below:

$$
\begin{aligned}
\frac{\partial F_{1}}{\partial k_{1}}= & f_{1}^{\prime}\left(k_{1}\right) g_{1}(\gamma)>0, \\
\frac{\partial F_{1}}{\partial \gamma}= & f_{1}\left(k_{1}\right) g_{1}^{\prime}(\gamma)<0, \\
\frac{\partial F_{2}}{\partial k_{1}}= & f_{p+1}\left(u_{p+1}(1)\right) g_{p+1}^{\prime}\left(\gamma-\int_{0}^{1} \frac{P(x)}{\sum_{i=1}^{m} e_{i}^{2} u_{i}} d x\right) \cdot \int_{0}^{1} \frac{P(x)\left(\sum_{i=1}^{m} e_{i}^{2} u_{i}\right)_{k_{1}}^{\prime} d x>0,}{\left[\sum_{i=1}^{m} e_{i}^{2} u_{i}(x)\right]^{2}} \\
\frac{\partial F_{2}}{\partial k_{p+1}}= & \left.f_{p+1}^{\prime}\left(u_{p+1}(1)\right) g_{p+1}\left(\gamma-\int_{0}^{1} \frac{P(x)}{\sum_{i=1}^{m} e_{i}^{2} u_{i}} d x\right) \cdot e^{-e_{p+1}(\phi(1)-\phi(0))}\right) \\
& +f_{p+1}\left(u_{p+1}(1)\right) g_{p+1}^{\prime}\left(\gamma-\int_{0}^{1} \frac{P(x)}{\sum_{i=1}^{m} e_{i}^{2} u_{i}} d x\right) \cdot \int_{0}^{1} \frac{P(x)\left(\sum_{i=1}^{m} e_{i}^{2} u_{i}\right)_{k_{p+1}}^{\prime}}{\left[\sum_{i=1}^{m} e_{i}^{2} u_{i}\right]^{2}}>0, \\
\frac{\partial F_{2}}{\partial \gamma}= & f_{p+1}\left(u_{p+1}(1)\right) g_{p+1}^{\prime}\left(\gamma-\int_{0}^{1} \frac{P(x)}{\sum_{i=1}^{m} e_{i}^{2} u_{i}} d x\right)>0, \\
\frac{\partial F_{3}}{\partial k_{1}}= & \sum_{e_{i}<0} \frac{d k_{i}}{d k_{1}}<0, \\
\frac{\partial F_{3}}{\partial k_{p+1}}= & \sum_{e_{i}>0} e_{i} \frac{d k_{i}}{d k_{p+1}}>0,
\end{aligned}
$$

where

$$
\begin{gathered}
\left(\sum_{i=1}^{m} e_{i}^{2} u_{i}\right)_{k_{1}}^{\prime}=\left(\sum_{e_{i}<0} e_{i}^{2} u_{i}\right)_{k_{1}}^{\prime}=\sum_{e_{i}<0} e_{i}^{2}\left(e^{-e_{i}(\phi(x)-\phi(0))}\right) \frac{d k_{i}}{d k_{1}}>0, \\
\left(\sum_{i=1}^{m} e_{i}^{2} u_{i}\right)_{k_{p+1}}^{\prime}=\left(\sum_{e_{i}>0} e_{i}^{2} u_{i}\right)_{k_{p+1}}^{\prime}=\sum_{e_{i}>0} e_{i}^{2}\left(e^{-e_{i}(\phi(x)-\phi(0))}\right) \frac{d k_{i}}{d k_{p+1}}>0 .
\end{gathered}
$$

The derivatives $d k_{i} / d k_{1}>0, d k_{i} / d k_{p+1}>0$ in (38) and (39) can be computed via (23) and (24), i.e.,

$$
\begin{gathered}
\frac{d k_{i}}{d k_{1}}=\frac{\left.a_{1} \cdot\left(g_{1}^{-1}\right)^{\prime}\right|_{\frac{a_{1}}{f_{1}\left(k_{1}\right)}} f_{1}^{\prime}\left(k_{1}\right) f_{i}^{2}\left(k_{i}\right)}{\left.a_{i} \cdot\left(g_{i}^{-1}\right)^{\prime}\right|_{\frac{a_{i}}{f_{i}\left(k_{i}\right)}} f_{i}^{\prime}\left(k_{i}\right) f_{1}^{2}\left(k_{1}\right)}, \quad i=2, \ldots, p, \\
\frac{d k_{i}}{d k_{p+1}}=e^{\left(e_{i}-e_{p+1}\right)(\phi(1)-\phi(0))} \frac{\left.a_{p+1}\left(g_{p+1}^{-1}\right)^{\prime}\right|_{\frac{a_{p+1}}{f_{p+1}\left(u_{p+1}(1)\right)}} f_{p+1}^{\prime}\left(u_{p+1}(1)\right) f_{i}^{2}\left(u_{i}(1)\right)}{\left.a_{i} \cdot\left(g_{i}^{-1}\right)^{\prime}\right|_{\frac{a_{i}}{f_{i}\left(u_{i}(1)\right)}} f_{i}^{\prime}\left(u_{i}(1)\right) f_{p+1}^{2}\left(u_{p+1}(1)\right)},
\end{gathered}
$$

$i=p+2, \ldots n$. We need to solve the $3 \times 3$ nonlinear system

$$
\mathbf{J}_{3 \times 3}\left(k_{1}, k_{p+1}, \gamma\right) \mathbf{Y}=-\mathbf{F}\left(k_{1}, k_{p+1}, \gamma\right)
$$

each time in order to solve the system (25)-(27). Here $\mathbf{Y}=\left[k_{1}, k_{p+1}, \gamma\right]^{\mathrm{T}}$ and $\mathbf{F}\left(k_{1}, k_{p+1}, \gamma\right)=\left[F_{1}, F_{2}, F_{3}\right]^{\mathrm{T}}$. 
The advantage of the algorithm, as one may notice, is that no matter how many species are in the electrochemical system, only a $3 \times 3$ nonlinear system (25)-(27) has to be solved each time, whereas in the conventional algorithm (finite difference scheme, for example), the dimension of the linear system to be solved increases drastically as the number of the species increases.

3.2. Numerical results and discussions. In the tests, we pick all source terms to be 1 for simplicity. Functions $g_{i}(x)=e^{E_{0}-x}-1, i=1, \ldots, p$, are chosen for the reacting species with negative charge; $g_{i}(x)=e^{x-E_{1}}-1, i=p+1, \ldots, n$, are chosen for the reacting species with positive charge, and functions $f_{i}(x)=x, i=1, \ldots, n$, unless otherwise stated. $E_{0}=1$ and $E_{1}=0$ are the given external electric potentials. Note that only the diffusion coefficient of the reacting species $\left(d_{i}, i=1, \ldots, n\right)$ and the total concentration of the inert species $\left(c_{i}, i=n+1, \ldots, m\right)$ need to be specified. The tolerance is set at $10^{-6}$ for all the tests. Profiles of concentration of each species $u_{i}$ and the electric potential $\phi$ for each test are drawn in Figures 3, 4, and 5 (see p. 524), respectively.

Test 1 . Four species in the solution for which two are reacting. Take $m=4, n=$ $2 ; e_{1}=-1, e_{2}=1, e_{3}=1, e_{4}=1 ; d_{1}=1, d_{2}=1 ; c_{5}=1, c_{6}=1 ;$ The initial values are $k_{1}=1.0, k_{p+1}=\max \left(\frac{m_{0}}{d_{p+1} e_{p+1}}, \sum_{i=n+1}^{m}\left|e_{i}\right| c_{i} e^{\left|e_{i}\right|\left(E_{0}-E_{1}\right)}\right)$ and $\phi(x)$ is a straight line $y=E_{1}+\left(E_{0}-E_{1}\right) x$. Here $u_{i}, i=1,2$ is the concentration of the reacting species. Then it takes 6 iterations to get the numerical solution. In this case (18) and (19) are not needed.

Test 2. Six species in the solution for which four are reacting. Take $m=6$, $n=4 ; e_{1}=-2, e_{2}=1, e_{3}=2, e_{4}=1, e_{5}=-1, e_{6}=2 ; d_{1}=3, d_{2}=1, d_{3}=$ $1.7, d_{4}=1 ; c_{5}=2, c_{6}=3 ; f_{1}(x)=x^{2}, f_{3}(x)=x^{3}, f_{4}(x)=x^{2}$; The initial values are $k_{1}=0.5, k_{p+1}=3$ and $\phi(x)$ is a straight line $y=E_{1}+\left(E_{0}-E_{1}\right) x$. Then it takes 8 iterations to compute the numerical solution. In this test, functions $f_{1}, f_{3}$, and $f_{4}$

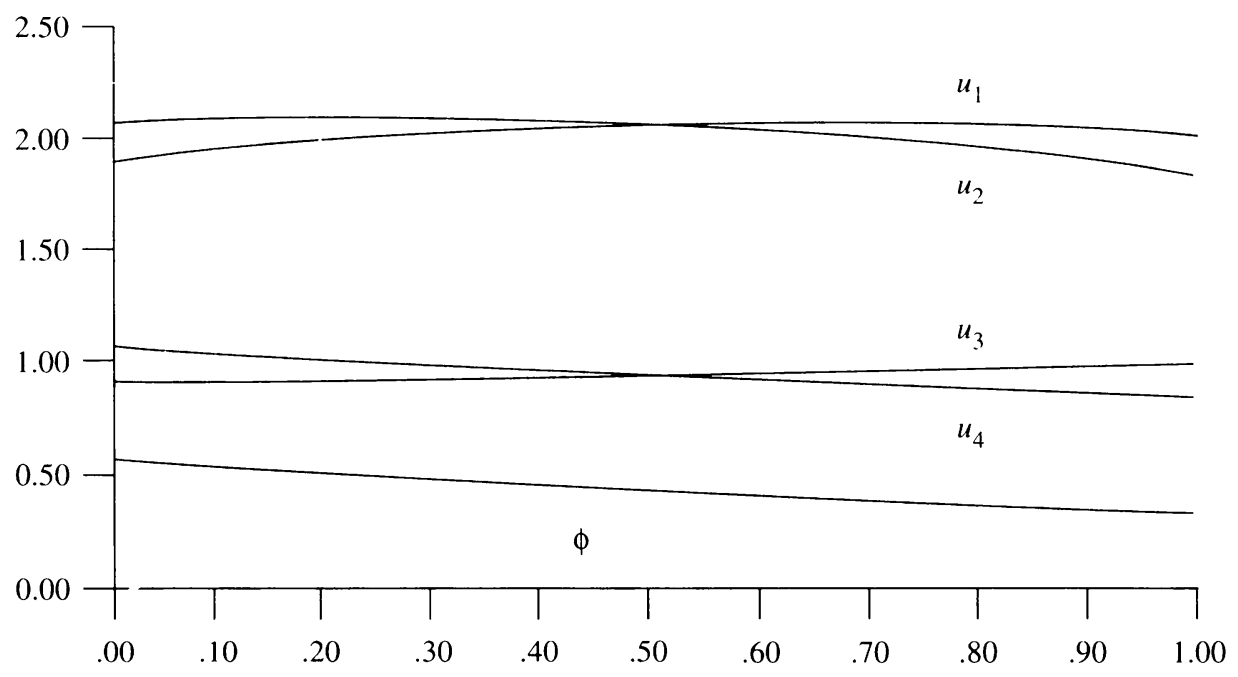

FIG. 3. Test 1. Two reacting species and two inert species 


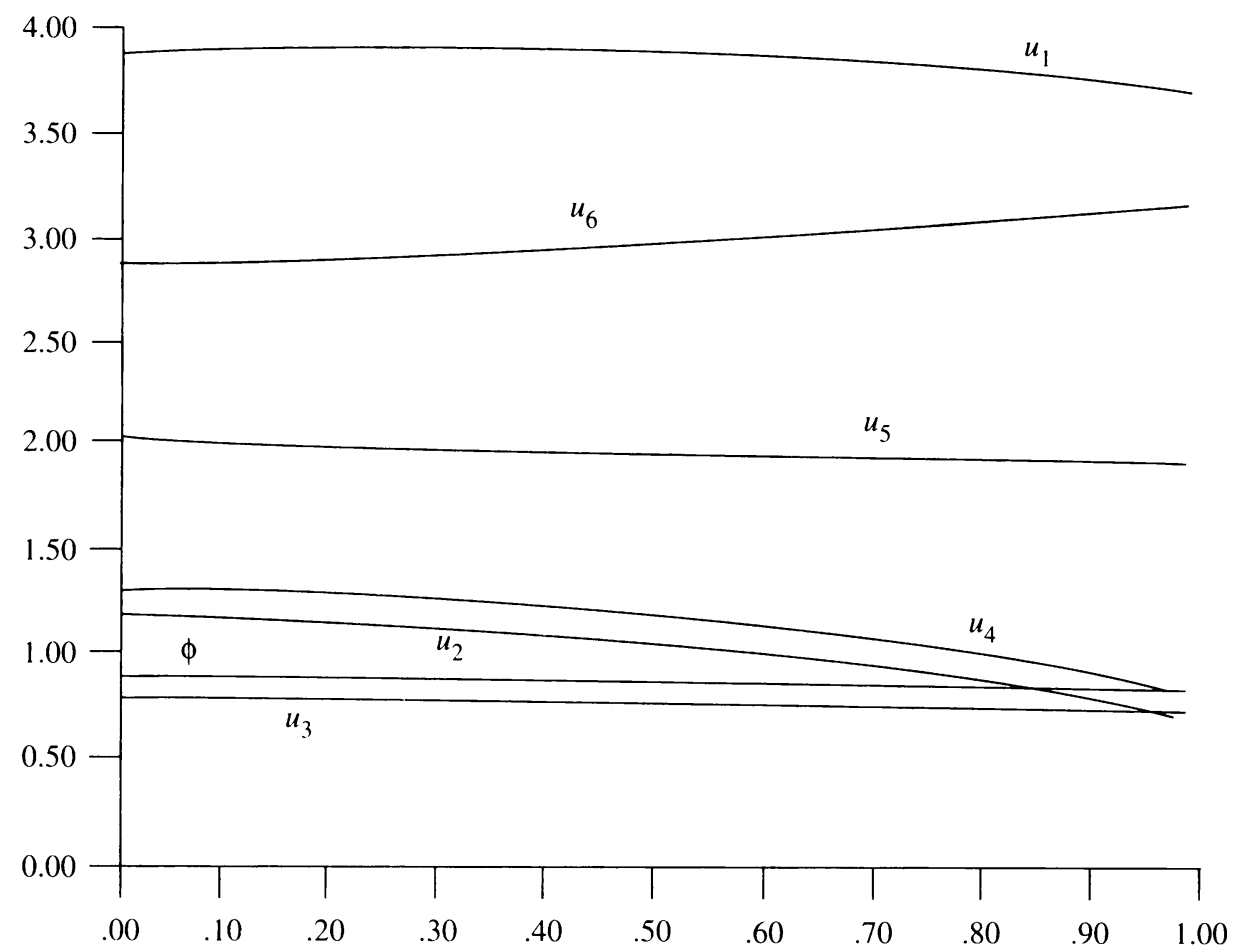

FIG. 4. Test 2. Four reacting species and two inert species

are not linear, which is usually the case in the kinetics expressions. $u_{i}, i=1, \ldots, 4$ are the concentrations of the reacting species and $r_{1}(x)=r_{2}(x)+r_{3}(x)+r_{4}(x)$, which is the case explained in the remark.

Test 3. Ten species in the solution for which six are reacting. Take $m=10$, $n=6 ; e_{1}=-1, e_{2}=0.7, e_{3}=1.3, e_{4}=1, e_{5}=-1.7, e_{6}=2.7, e_{7}=-1, e_{8}=$ $5, e_{9}=-2.5, e_{10}=0.3 ; d_{1}=1, d_{2}=4, d_{3}=1.4, d_{4}=1, d_{5}=1.5, d_{6}=2 ; c_{7}=$ $2.7, c_{8}=1.7, c_{9}=0.4, c_{10}=3 ; f_{1}(x)=x^{2}, f_{3}(x)=x^{3}$. The initial values are $k_{1}=$ $0.5, k_{p+1}=4$ and $\phi(x)$ is a straight line connecting points $\left(0, E_{0}\right)$ and $\left(1, E_{1}\right)$. It takes 8 iterations to compute the numerical solution. In this test, functions $f_{1}$ and $f_{3}$ are not linear. $u_{i}, i=1, \ldots, 6$ are the concentrations of the reacting species.

It is noted that the concentration of inert species with positive charge is always increasing and that with negative charge is decreasing. This is a direct result of (11) and the fact that $u_{i}>0$ and $d \phi / d x<0, i=n+1, \ldots, m$. For each reacting species, $\left.\frac{d u_{i}(x)}{d x}\right|_{x=0}>0, i=p+1, \ldots, n$ and $\left.\frac{d u_{i}(x)}{d x}\right|_{x=1}<0, i=1, \ldots, p$. Since

$$
\frac{d u_{i}(x)}{d x}=-e_{i} \frac{d \phi(x)}{d x} u_{i}(x)-\frac{1}{d_{i} e_{i}} p_{i}(x), \quad i=1,2, \ldots, n,
$$

it can be checked that if $e_{i}<0$,

$$
\left.\frac{d u_{i}(x)}{d x}\right|_{x=1}=-\left.e_{i}\left[\frac{d \phi(x)}{d x} u_{i}(x)\right]\right|_{x=1}-\left.\frac{1}{d_{i} e_{i}} p_{i}(x)\right|_{x=1}<0
$$




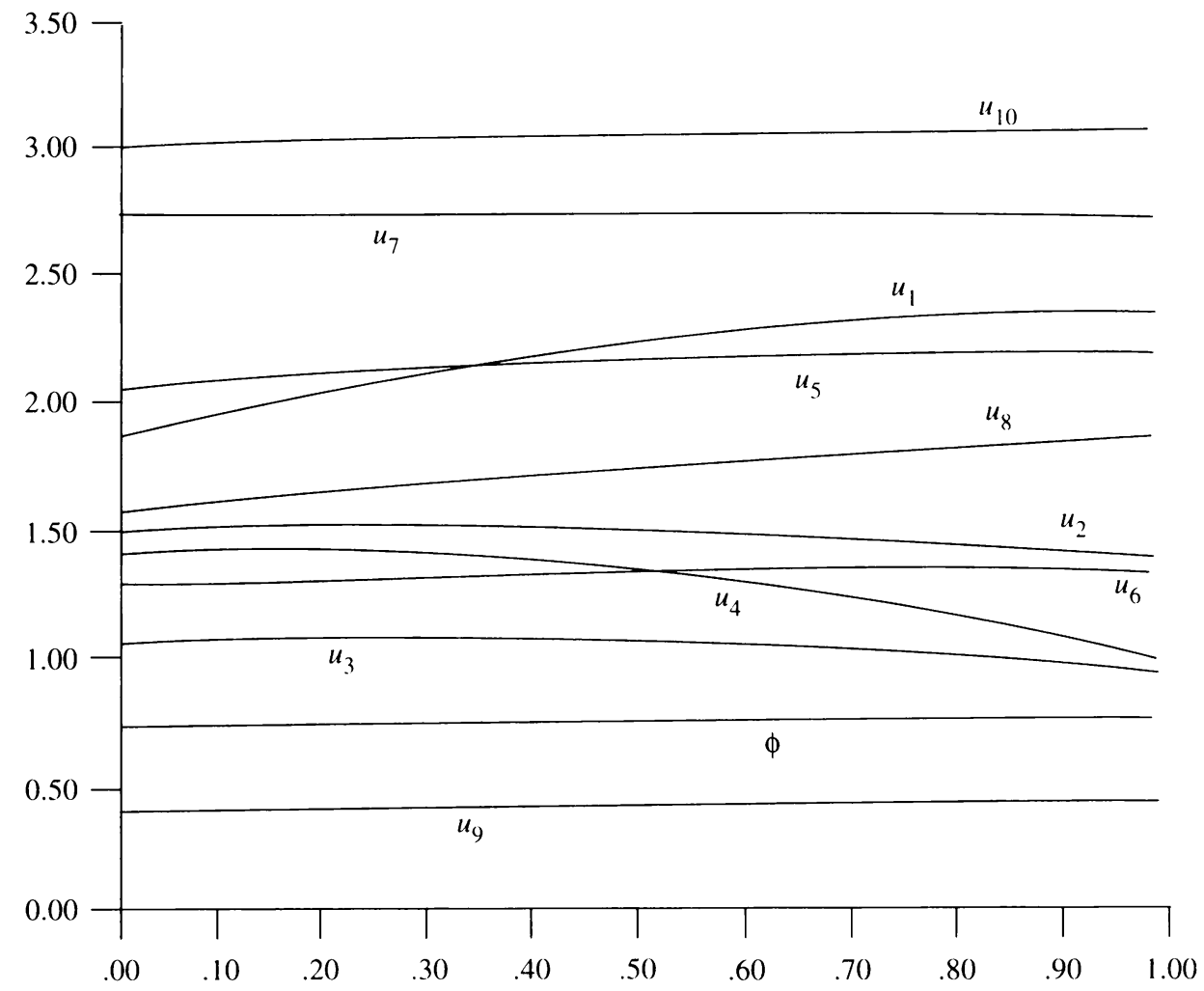

FIG. 5. Test 3. Six reacting species and four inert species

because $p_{i}(1)=0, i=1, \ldots, p$. Similarly, if $e_{i}<0$,

$$
\left.\frac{d u_{i}(x)}{d x}\right|_{x=0}=-\left.e_{i}\left[\frac{d \phi(x)}{d x} u_{i}(x)\right]\right|_{x=0}-\left.\frac{1}{d_{i} e_{i}} p_{i}(x)\right|_{x=0}>0
$$

because $p_{i}(0)=0, i=p+1, \ldots, n$.

4. Conclusion. Our proof not only guarantees the existence of the steady state solution for this electrochemical process, but also gives rise to a robust numerical algorithm to solve the system with nonlinear nonlocal boundary conditions. The algorithm is more efficient and faster than the conventional finite difference methods or those computational algorithms used in electrochemistry (most of them are modified finite difference methods).

\section{REFERENCES}

[1] Y. S. Choi and Roger Lui, Uniqueness of steady-state solutions for an electrochemistry model with multiple species, J. Differential Equations 108 2, 424-437 (1994)

[2] Y. S. Choi and Kwong-Yu Chan, A parabolic equation with nonlocal boundary conditions arising from electrochemistry, J. Nonlinear Analysis Theory, Methods and Applications 18, No. 4, 317-331 (1992)

[3] Y. S. Choi and Kwong-Yu Chan, Exact solution of transport in a binary electrolyte, J. Electroanalytical Chemistry and Interfacial Electrochemistry 334, 13-23 (1992) 
[4] Y. S. Choi and Roger Lui, Analysis of electrochemistry model with zero-flux boundary conditions, Applicable Analysis 49, Nos. 3-4, 277-288 (1993)

[5] Y. S. Choi and Xun Yu, Steady state solution for electroplating, IMA J. Appl. Math. 51, No. 3, 251-267 (1993)

[6] P. Delahay and C. W. Tobias, Advances in Electrochemistry and Electrochemical Engineering, Vol. 5, John Wiley and Son, 1967

[7] P. C. Fife, O. A. Palusinski, and Y. Su, Electrophoretic traveling waves, Trans. Amer. Math. Soc. 310, 759-780 (1988)

[8] D. Gilbarg and N. S. Trudinger, Elliptic Partial Differential Equations of Second Order, Second Edition, Springer-Verlag, 1983

[9] John S. Newman, Electrochemical Systems, Prentice-Hall, Inc., N.J., 1973

[10] Herbert Amann, Reaction-diffusion problems in electrolysis, Nonlinear Differential Equations and Applications 1, No. 1, 91-117 (1994) 